\title{
A Modified CDC Biofilm Reactor to Produce Mature Biofilms on the Surface of PEEK Membranes for an In Vivo Animal Model Application
}

\author{
Dustin L. Williams • Kassie L. Woodbury • \\ Bryan S. Haymond · Albert E. Parker · \\ Roy D. Bloebaum
}

Received: 29 November 2010/ Accepted: 17 February 2011/Published online: 25 March 2011

(C) Springer Science+Business Media, LLC (outside the USA) 2011

\begin{abstract}
Biofilm-related infections have become a major clinical concern. Typically, animal models that involve inoculation with planktonic bacteria have been used to create positive infection signals and examine antimicrobial strategies for eradicating or preventing biofilm-related infection. However, it is estimated that $99.9 \%$ of bacteria in nature dwell in established biofilms. As such, open wounds have significant potential to become contaminated with bacteria that reside in a well-established biofilm. In this study, a modified CDC biofilm reactor was developed to repeatably grow mature biofilms of Staphylococcus aureus on the surface of polyetheretherketone (PEEK) membranes for inoculation in a future animal model of orthopaedic implant biofilm-related infection. Results indicated that uniform, mature biofilms repeatably grew on the surface of the PEEK membranes.
\end{abstract}

Keywords Biofilm · Modified $\cdot$ Reactor $\cdot$ CDC . Membrane $\cdot$ Repeatability

D. L. Williams · R. D. Bloebaum ( $\square)$

Department of Bioengineering, University of Utah,

Salt Lake City, UT, USA

e-mail: roy.bloebaum@hsc.utah.edu

D. L. Williams

e-mail: dustin.williams@utah.edu

K. L. Woodbury · B. S. Haymond · R. D. Bloebaum Department of Veterans Affairs, Bone and Joint Research Laboratory (151F), Salt Lake City, UT, USA

\section{A. E. Parker}

Center For Biofilm Engineering, Montana State University, Bozeman, MT, USA

R. D. Bloebaum Department of Orthopaedics, University of Utah School of Medicine, Salt Lake City, UT, USA

\section{Introduction}

Biofilms have become a major research interest in recent years. For example, the role of biofilms in chronic orthopaedic device-related infections has been particularly concerning as highlighted by the failed attempts of orthopaedic surgeons to treat patients who are adversely affected by biofilm formation on and near orthopaedic devices $[3,5,16]$. These patients often require expensive and compromising revision surgery, implant removal or, in a worst case scenario, amputation. Death can also be a devastating outcome. Biofilm-related infections similarly affect injured military personnel, in particular, those suffering from severely contaminated blast injuries in the current Afghanistan and Iraq conflicts [13, 14].

In an attempt to prevent these biofilm-related infections from developing near or on orthopaedic devices, animal models have been used to create positive infection signals and to examine antimicrobial strategies for treatment $[4,7,8,17]$. Typically, these animal models have consisted of implanting a device in conjunction with inoculation of planktonic (freefloating) bacteria near the device. The expectation has either been that planktonic cells will form a biofilm on or near the implant and result in biofilm-related infection, or that planktonic cells alone will develop infection.

However, since the 1978 hypothesis of Costerton et al. [6] that bacteria in nature preferentially aggregate in biofilms, it has been estimated that approximately $99.9 \%$ of bacteria in nature dwell in biofilms [11, 12]. Moreover, Bakken [1] has shown that $1 \mathrm{~g}$ of fertile soil can contain up to 5 billion bacterial cells. If contaminated with even 1 or $2 \mathrm{~g}$ of soil, it is plausible that open fractures or other types of open wounds may have significant contamination with very high inocula of bacteria residing in well-established biofilms. Therefore, in vivo models of infection that 
involve the use of well-established biofilms may contribute to our understanding of biofilm-related infection as well as methods of safely eradicating the biofilm and related infection without surgery and implant removal.

In this study, a modified CDC biofilm reactor was developed to grow well-established Staphylococcus aureus biofilms on the surface of polyetheretherketone (PEEK) membranes. It is intended for these biofilm-ridden membranes to be used to inoculate future animal models of biofilm-related infection, pending confirmation that mature, uniform and viable biofilms can be repeatably formed on the PEEK membranes.

\section{Materials and Methods}

\section{Isolate Selection}

A freshly cultured, clinical isolate of methicillin-resistant S. aureus (MRSA) was collected from a patient and used for this study. More specifically, a patient who underwent knee surgery developed infection and an aspirate was sent to ARUP Laboratories, Salt Lake City, UT for characterization and susceptibility testing. The isolate was characterized as MRSA based on resistance to penicillin and oxacillin using the Kirby Bauer disc diffusion technique. Preliminary work indicated that the isolate was a biofilm former following black colony formation on Congo Red agar and detection of the icaADBC gene cluster. When grown as a biofilm and used as initial inocula, biofilms of this strain caused osteomyelitis in a pilot animal model of biofilm implant-related infection (unpublished data).

\section{Modified Design of the CDC Biofilm Reactor}

The CDC biofilm reactor was purchased from Biosurface Technologies (Bozeman, MT). The original design of the reactor is provided in Fig. 1a. A modified lid was designed and machined using a local machine shop. The modified lid contained four slots into which guillotine-like holders (20 $\mathrm{cm}$ in length with a $3 \mathrm{~mm}$ groove down the middle of the interior portion) made of ultra-high-molecular-weightpolyethylene (UHMWPE) were inserted (Fig. 1b). Into the guillotine holders were placed PEEK membranes that were held in place between two 316 stainless steel plates with a $0.64 \mathrm{~cm}^{2}$ opening (Fig. 1b). A photograph of the modified reactor is provided in Fig. 1c. All other aspects of the reactor were the same as the original CDC biofilm reactor.

\section{Biofilm Growth in Reactor}

The modified reactor held eight PEEK membranes with two membranes in each of the four guillotine holders (see
Fig. 1). PEEK membranes were first sonicated for $10 \mathrm{~min}$ in detergent, rinsed under running reverse osmosis water for $10 \mathrm{~min}$, sonicated in reverse osmosis water for $10 \mathrm{~min}$ and rinsed once again using $70 \%$ ethanol. The reactor was then assembled and all components autoclaved before use.

Following American Society for Testing and Materials (ASTM) standard E2562-07, the modified reactor was run under the following conditions: approximately $1.5 \times 10^{8}$ bacterial cells were inoculated into $500 \mathrm{ml}$ of brain heart infusion (BHI) broth (modified) in the biofilm reactor. The rotator was stirred at $130 \mathrm{rpm}$, and the unit was placed in an incubator set at $28.5^{\circ} \mathrm{C}$ for $24 \mathrm{~h}$. A $10 \%$ BHI broth (modified) solution was then flowed through the reactor at $6.94 \mathrm{ml} / \mathrm{min}$ for $24 \mathrm{~h}$.

Sample Fixation, Dehydration and Imaging

To qualitatively observe the biofilms that had developed, eight membranes were imaged using SEM. For imaging preparation, membranes were removed from the reactor, placed in $2.5 \%$ glutaraldehyde for $24 \mathrm{~h}$ and then dehydrated using ascending grades of ethanol with $3 \times 20 \mathrm{~min}$ exchanges of each up to $100 \%$. Samples were dried in a desiccator overnight, sputter coated with $\sim 3 \mathrm{~nm}$ of gold, and imaged using a FEI NOVANano SEM 600.

On two membranes, the Filmtracer ${ }^{\mathrm{TM}}$ LIVE/DEAD ${ }^{\circledR}$ stain (Invitrogen, Carlsbad, CA) was used in conjunction with confocal laser scanning microscopy (CLSM) to qualitatively observe the degree of live versus dead bacteria on the membrane. The biofilms were observed using a FV-1000 XY inverted CLSM microscope with a $60 \times$ water objective.

\section{Bacterial Quantification}

In order to determine uniformity, following eight separate runs of the modified biofilm reactor, a total of 64 PEEK membranes were used to quantify the number of bacteria that grew on the surface of each membrane. The membranes from each run were randomly assigned to one of two treatment groups (i.e., with four membranes per group).

Following growth in the reactor, the four membranes in Group 1 were removed from the guillotine holders, rinsed $3 \times$ in $6 \mathrm{ml}$ of saline, placed on a shaker at $100 \mathrm{rpm}$ for $20 \mathrm{~min}$ and allowed to remain stationary for $1.5 \mathrm{~h}$. The membranes were then vortexed for $1 \mathrm{~min}$, sonicated at $47 \mathrm{kHz}$ and $1.8 \mathrm{~W} / \mathrm{cm}^{2}$ for $10 \mathrm{~min}$, allowed to recover from sonication for $20 \mathrm{~min}$ and then enumerated using a 10-fold dilution series. $100 \mu \mathrm{l}$ of each dilution $(1: 10-1: 10,000,000)$ was plated onto Columbia blood agar and incubated overnight at $37^{\circ} \mathrm{C}$. The following day, the colony forming units (cfu) were counted to determine the number of bacteria per membrane (cfu/membrane). Fifteen randomly selected membranes were imaged by 
Fig. 1 a Schematic diagram of the CDC biofilm reactor. b Schematic diagram of the modified CDC biofilm reactor lid and guillotine-like holder. c Photograph of the completed modified CDC biofilm reactor

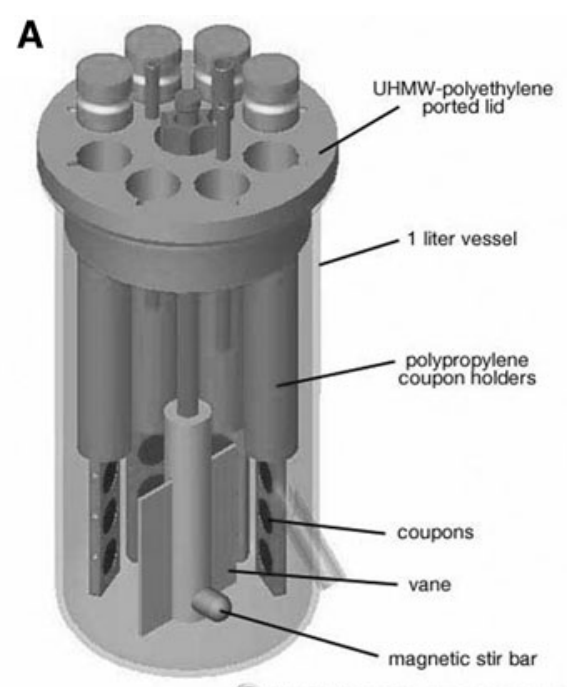

(2) Blosutiace Tedundogles tha.

B
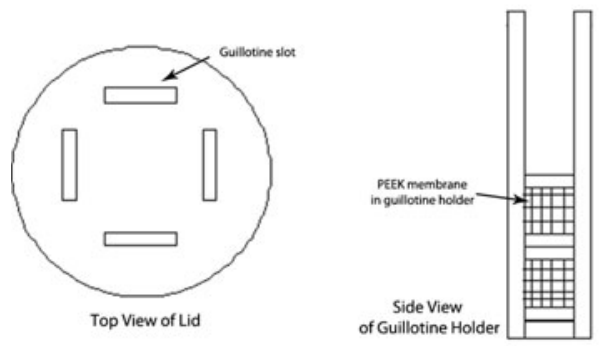

SEM to confirm that bacteria had been removed from the surface.

The rationale for shaking the membranes at $100 \mathrm{rpm}$ for 20 min was to model the car ride that they will be exposed to when they are transported from the lab to the site of animal surgery. Furthermore, the four membranes in Group 1 were allowed to remain stationary for $1.5 \mathrm{~h}$ to model the time that they will be stationary whilst the surgical facility and animals are prepped for surgery before inoculation. Group 2 membranes were treated in the same manner as those in Group 1 with the exception that they were allowed to remain stationary for $3 \mathrm{~h}$ as opposed to $1.5 \mathrm{~h}$ so as to model the time that they will remain stationary during the first surgery of the future animal model.

The density of bacteria in cfu/membrane was recorded for each PEEK membrane and then $\log _{10}$ transformed. For each of the two treatment groups separately, an ANOVA was fit, with run as a random effect, to calculate the mean and repeatability standard deviation (SD) of the log densities on the PEEK membranes over the eight reactor runs. 
Another ANOVA, with group as a fixed effect and run as a random effect, was used to compare the group means and to calculate the repeatability SD for both groups pooled together. These statistics were used to determine if any difference in bacterial response could be seen between membranes that were allowed to remain in a dilute broth solution for varying periods of time. The analyses also showed the percentage of the repeatability variance attributable to within- and between-run sources.

\section{Results}

Growth on PEEK Membranes Within the Biofilm Reactor

Images collected of the PEEK membranes following growth, sample fixation and dehydration indicated that copious amounts of biofilm formed on the membrane surfaces (Fig. 2a). Furthermore, each of the three indicators
Fig. 2 a Representative SEM image of the $S$. aureus biofilm. b Additional image of the EPS providing a scaffold for bacterial cells within the biofilm to create a bridge from one surface of the PEEK membrane to another. c Representative image showing a threedimensional structure of the biofilm extending vertically from the surface of the PEEK membrane. d Representative image showing possible water channels within the biofilm structure (arrow). e The LIVE/ $\mathrm{DEAD}^{\circledR}$ stain showed living cells as green and dead cells as red. This image shows a representative $\mathrm{Z}$ projection slice of the $S$. aureus biofilm collected using CLSM and indicates that the cells within the biofilm were predominantly living with an estimated ratio of live to dead cells being 1000:1. Original magnification was $\times 60$ (color images can be seen in the online version or upon request to the authors)
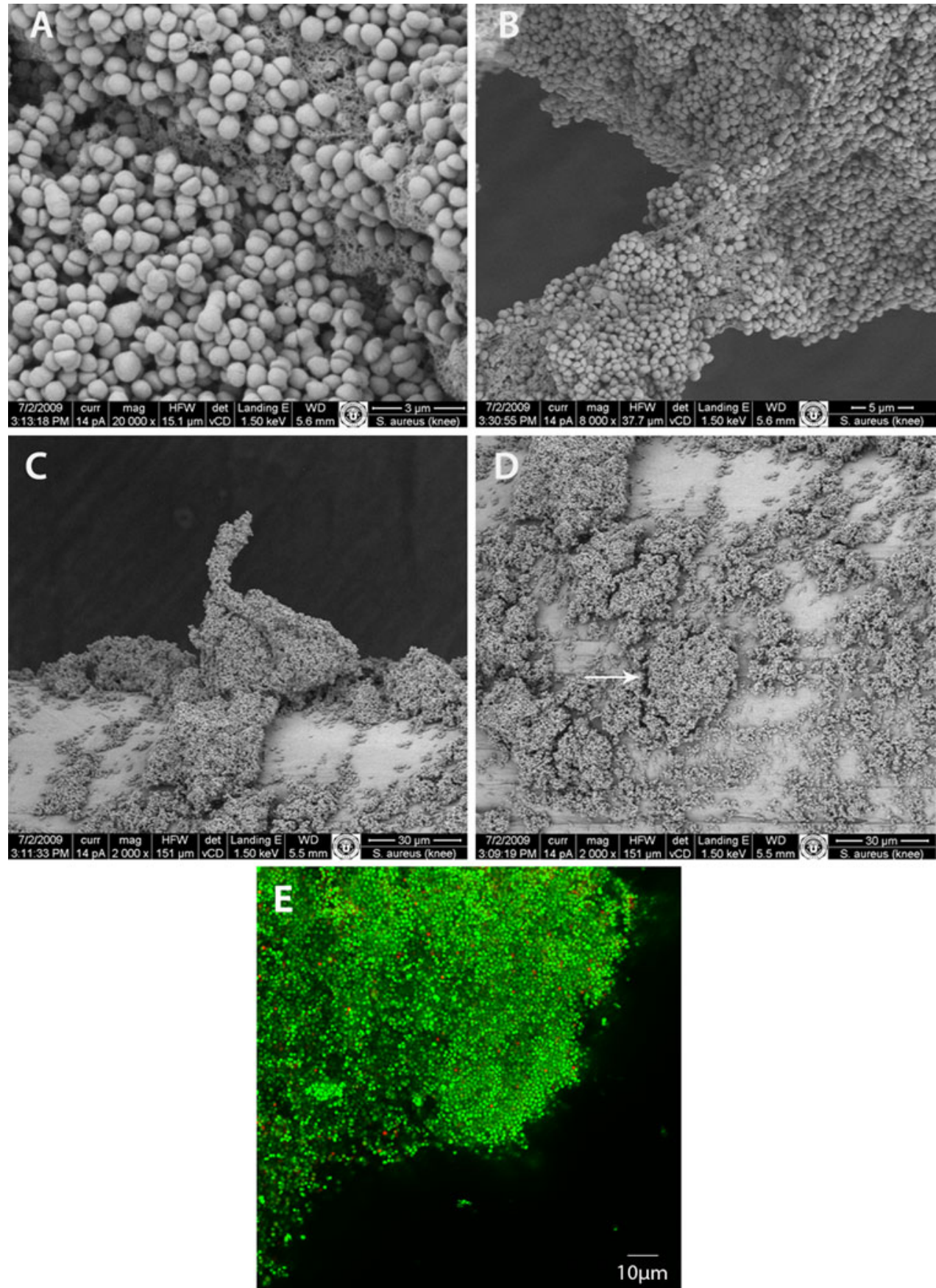
that a mature biofilm had developed were detected [10], i.e., significant EPS production (Fig. 2a and b), threedimensional structures of mushroom- or pillar-like formations (Fig. 2c) and possible water channel development (Fig. 2d) were observed upon SEM analysis. In addition, the EPS matrix appeared to act as a scaffold to which the bacteria attached to create a "bridge" that connected cells of the biofilm from one strand of the PEEK membrane to another (Fig. 2b). These results indicate that mature biofilms did form on the surface of PEEK membranes when grown within the modified membrane reactor.

Notably, although the temperature of the incubator was set at $28.5^{\circ} \mathrm{C}$, the recorded temperature of the broth after the first $24 \mathrm{~h}$ of each run was $28.1 \pm 1.2$. After $48 \mathrm{~h}$ of growth, the broth temperature from each run was $29.8 \pm 0.5$.

\section{Bacterial Quantification}

The number of bacteria on each PEEK membrane was quantified following eight separate runs of the reactor, with eight membranes per run, and four membranes randomly assigned to each of two treatment groups to confirm uniformity. The data are shown in Fig. 3.

Mean $\log$ densities and repeatability SDs for each of the two groups individually and also for all 64 membranes pooled together are given in Table 1. The table corroborates what is evident in Fig. 3: the two groups have similar means and repeatability SDs. A 90\% confidence interval for the difference of the Group 1 mean $\log$ density subtracted from the Group 2 mean log density was found to be $(0.06,0.19)$, which indicates statistical equivalence between the two group means at a significance level of 5\% as long as mean differences up to 0.19 are considered to be negligible.

Taken together, these results show that copious amounts of $S$. aureus biofilm formed on the surface of each PEEK membrane within the reactor and that the mean log densities for the two treatment groups were statistically equivalent. Furthermore, the biofilm log density exhibited acceptable repeatability from run to run under the conditions described.

SEM images of the PEEK membranes that were collected after the quantification process indicated that only
Fig. 3 An individual value plot of the log density of bacteria per PEEK membrane. Group 1 (open circle) membranes were those that were allowed to remain stationary for $1.5 \mathrm{~h}$ before quantification. Group 2 (open square) membranes were those that were allowed to remain stationary for $3 \mathrm{~h}$ before quantification. Each point represents one membrane

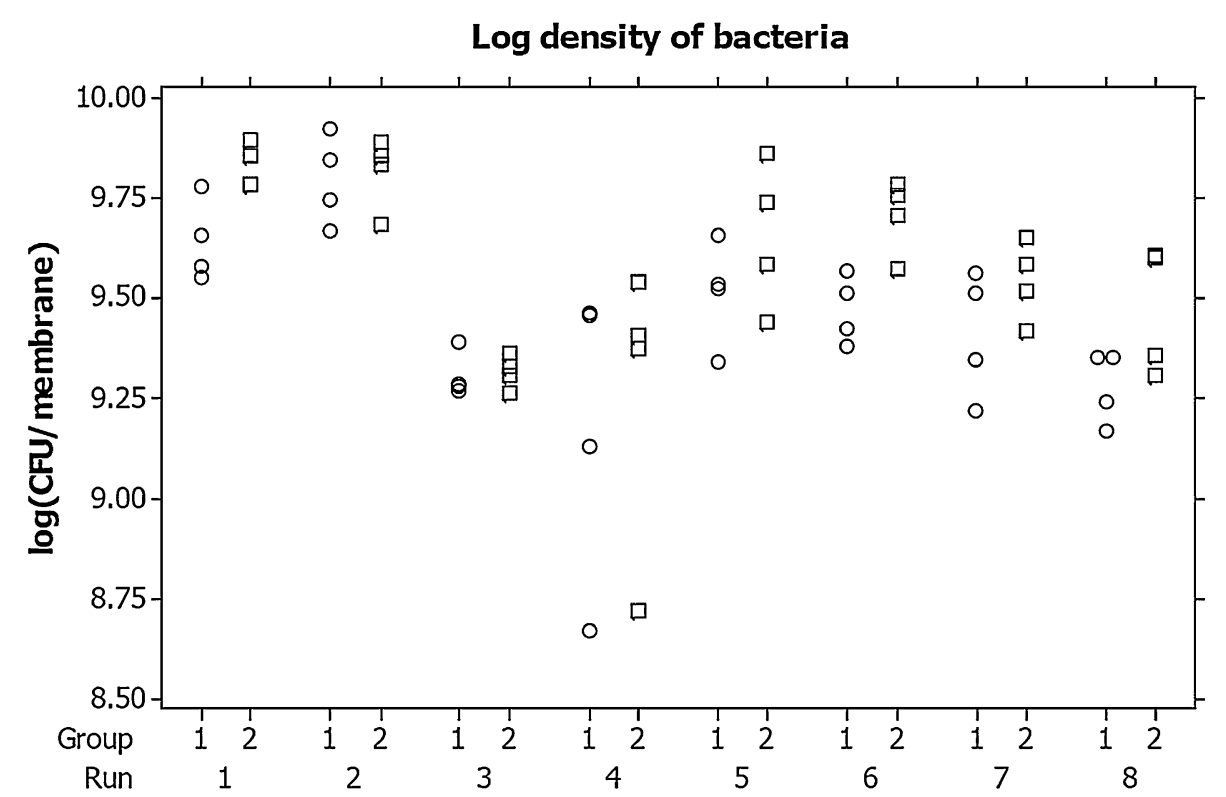

Table 1 Statistical characteristics for each of the two treatment groups, and both groups pooled together

\begin{tabular}{llllll}
\hline Group & Mean & SE & Repeatability SD & \multicolumn{2}{l}{ Percentage contribution } \\
\hline 1 & & & Within run $(\%)$ & 17 & 83 \\
2 & 9.45 & 0.0296 & 0.2020 & 15 & 85 \\
Both 1 and 2 & 9.58 & 0.0297 & 0.2162 & 14 & 86 \\
\hline
\end{tabular}

The standard error (SE) of the mean and the repeatability SD are based on four membranes per run. These results demonstrate the uniformity of the biofilm formation on the PEEK membranes 
sparse microcolonies of bacteria remained on the surface. As such, the number of bacteria in the microcolonies was inconsequential with respect to the billions of cells that were enumerated (SEM images not shown).

\section{Discussion}

An increased understanding of the important role of biofilms in device-related and chronic infections suggests that in vitro and in vivo studies may be strengthened with the use of mature biofilms as opposed to the application of planktonic bacteria alone. This study highlighted the ability of the CDC biofilm reactor to be modified in such a way that mature biofilms could be grown on a biocompatible polymer surface that can later be applied towards in vivo experimentation. Taken together, the hypothesis that mature biofilms would develop on the surface of PEEK membranes in a repeatable fashion was supported.

Interestingly, during the optimization of the modified reactor, it was found that when the unit was placed in an incubator set at $37^{\circ} \mathrm{C}$, biofilms did not form as well as when they were grown at $28.5^{\circ} \mathrm{C}$. More specifically, when grown at $37^{\circ} \mathrm{C}$, there was no biofilm growth on the PEEK membranes by visual observation, whereas at $28.5^{\circ} \mathrm{C}$, copious amounts of biofilm could be seen. Since our group was interested in having $\sim 10^{9}$ cells/membrane, no further runs at $37^{\circ} \mathrm{C}$ were performed. Nevertheless, this finding was similar to that of Rode et al. [15]. They found that several clinical and food-related isolates of $S$. aureus produced biofilms optimally near $30^{\circ} \mathrm{C}$.

The overall mean bacterial $\log$ density was $9.51 \mathrm{log}$ (cfu/membrane), and the repeatability SD was 0.2130 . As a comparison, in the 13 experiments described by Buckingham-Meyer et al. [2], S. aureus ATCC 6538 biofilm grown in the CDC reactor on glass coupons exhibited a mean of $8.3 \log \left(\mathrm{cfu} / \mathrm{cm}^{2}\right)$ and a repeatability SD of 0.224 (based on two coupons). In a different set of 12 experiments, Buckingham-Meyer et al. [2] grew Pseuodomonas aeruginosa biofilm in the CDC reactor on glass coupons, with a mean of $8.5 \log \left(\mathrm{cfu} / \mathrm{cm}^{2}\right)$ and a repeatability SD of 0.211 . Goeres et al. [9] grew P. aeruginosa biofilm in the CDC reactor on polycarbonate coupons over nine experiments and reported a mean of $7.06 \log \left(\mathrm{cfu} / \mathrm{cm}^{2}\right)$ and a repeatability SD of 0.510 (based on two coupons per experiments). Thus, the biofilm bacteria grown on PEEK membranes in the modified CDC reactor exhibited acceptable run to run repeatability in our study.

Demonstrating that biofilm growth on the PEEK membranes was repeatable from run to run was an important aspect of this project. More specifically, if these biofilms of $S$. aureus on PEEK membranes are to be used in future animal models, it is important that individual animals receive statistically equivalent biofilm challenges. Therefore, the statistical equivalence of the biofilm grown on membranes in each of the treatment groups suggests that these biofilms will be used reliably and repeatably in a future animal model of device-related infection.

Acknowledgments This material is based upon study supported by the Office of Research and Development, Rehabilitation R\&D Service, Department of Veterans Affairs, Salt Lake City, UT. The project described was also supported by Award Number R01AR057185 from the National Institute Of Arthritis And Musculoskeletal And Skin Diseases. The content is solely the responsibility of the authors and does not necessarily represent the official views of the National Institute Of Arthritis And Musculoskeletal And Skin Diseases or the National Institutes of Health. This study was also supported by the Albert and Margaret Hofmann Chair and the Department of Orthopaedics, University of Utah School of Medicine, Salt Lake City, UT. The authors also acknowledge the help of Brad Isaacson, $\mathrm{PhD}$ for his contributions to the design of the modified CDC biofilm reactor and Dennis Romney for machining the modified reactor parts.

\section{References}

1. Bakken LR (1985) Separation and purification of bacteria from soil. Appl Environ Microbiol 49:1482-1487

2. Buckingham-Meyer K, Goeres DM, Hamilton MA (2007) Comparative evaluation of biofilm disinfectant efficacy tests. J Microbiol Methods 70:236-244

3. Campoccia D, Montanaro L, Arciola CR (2006) The significance of infection related to orthopedic devices and issues of antibiotic resistance. Biomaterials 27:2331-2339

4. Chou TG, Petti CA, Szakacs J et al (2010) Evaluating antimicrobials and implant materials for infection prevention around transcutaneous osseointegrated implants in a rabbit model. J Biomed Mater Res A 92:942-952

5. Costerton JW (2005) Biofilm theory can guide the treatment of device-related orthopaedic infections. Clin Orthop Relat Res 437:7-11

6. Costerton JW, Geesey GG, Cheng KJ (1978) How bacteria stick. Sci Am 238:86-95

7. Darouiche RO, Mansouri MD, Zakarevicz D et al (2007) In vivo efficacy of antimicrobial-coated devices. J Bone Joint Surg Am 89:792-797

8. Darouiche RO, Mansouri MD, Gawande PV et al (2009) Antimicrobial and antibiofilm efficacy of triclosan and Dispersin B combination. J Antimicrob Chemother 64:88-93

9. Goeres DM, Loetterle L, Hamilton MA et al (2005) Statistical assessment of a laboratory method for growing biofilms. Microbiology 151:757-762

10. Jiang X, Pace JL (2006) Microbial biofilms. In: Pace JL, Rupp ME, Finch RG (eds) Biofilms infection and antimicrobial therapy. Taylor and Francis Group, Boca Raton, p 4

11. Wimpenny J, Manz W, Szewzyk U (2000) Heterogeneity in biofilms. FEMS Microbiol Rev 24:661-671

12. Murphy TF, Kirkham C (2002) Biofilm formation by nontypeable Haemophilus influenzae: strain variability, outer membrane antigen expression and role of pili. BMC Microbiol 2:7

13. Murray CK (2008) Epidemiology of infections associated with combat-related injuries in Iraq and Afghanistan. J Trauma 64:S232-S238

14. Owens BD, Kragh JF Jr, Macaitis J et al (2007) Characterization of extremity wounds in operation Iraqi freedom and operation enduring freedom. J Orthop Trauma 21:254-257 
15. Rode TM, Langsrud S, Holck A et al (2007) Different patterns of biofilm formation in Staphylococcus aureus under food-related stress conditions. Int J Food Microbiol 116:372-383

16. Taubes G (2008) The bacteria fight back. Science 321:356-361
17. Williams D, Bloebaum R, Petti CA (2008) Characterization of Staphylococcus aureus strains in a rabbit model of osseointegrated pin infections. J Biomed Mater Res A 85:366-370 